\title{
Members' Page/Page des membres
}

New Members for the year 2000

Dan Adams

Peter Affleck

Brian Allen

Kevin Allen

Colin H. Anderson

Nathan Antoniuk

Thomas Archibald

Geoff Atkinson

Christen Audet

Andre Bachman David Bain

Richard Bambrick

Lindsay Banks

Adam M. Barkovitz

Edward R. Barnett

Shawn Barraclough

David Bartesko

Lee Barton

Darren Bath

Darrell Beam

Bryan Beaton

Nadyre Beaulieu

Norman Begin

Greg Behuniak

John Belanger

Keith Beraska

Dan Berry

Ken Birkett

William A Black

William M Black

Steve Blanton

Katherine Bleiker

Brent Bochon

Victor Boisvert

Peter Bontkes

Valerie Bourgon Hugh Boyd

Amanda Brackenreed John Brewer

Ellen Brezina

Sarah Jane Bros

Mark J.H. Brown Jeff Brown

Dave Michael Brown

Andrew Brown

Darren Brownlie John Bruce

Robert Michael Buck

G.A. (Tony) Bull

Dale Bullock

Richard H. Butt

Sofie L Caap

Elisha Cahoon

Isabelle Camirand

Colin Campbell

Keith Campbell

Roy Campbell

Donald Carr

Amy Carriere

Stuart Carter

Giuliana Casimirri

David J. Chapeskie

Keith Chapman

Tom Charles

Suzelin Chew

Eric Chiasson

Mark Chileen

Murray P. Chomokovski

Mary-Marie Christensen
Garth Christensen

Mecailla F. Christensen

Mathew G. Christie

Robert J Churma

Mike A. Cloet

Raymond Cloutier Dave Coish

Geoffery C. Cole

Barry J. Coleman

Spooner Collins

John Collins

Martin Cooke

Carl Corbett

Anita Cote

Brian Cote

Valerie Courtois

Tracey Cove

Dennis Cox

Christine Cripps-Woods

Wendy Crosina

Gordon Crowder

Chris Culbert

Gregory Cunliffe

James Cuthbertson

Stacy Cuzzocrea

Mark A. Dadebo

Crescentia Dakubo

Brian Davies

Cory Davis

Robert Davis

Sam M. Davis

Tammy De Costa

Damon Deacon

Theresa L. DeLaronde

Mary Margaret Delury

Emile Desnoyers

Stephen J. Dika

Darren Dillenbeck Steve Dodds

Aaron Doepel

Carol Doering

Kathleen Doerkson

Cynthia Doiron

C. Sean Dolter

Teresa Domingues

Bradley Donkin

Connie Down-Cicoria

Martha G.M. Dowsley

Dennis Driscoll Kelly Drolet

Phillipe L. Dube

George Duffy

Ian Dunk

Robert Dunn

James Garfield Dunn

Sherri Dunne

Suzanne L. Dupuis

Eric H. Earle

Jason Easton

Michael E Elfstedt

Barry C. Elkins

Bart Elliott

Elizabeth Ellis

Bradley Epp

Darcy Evanochko Robin Fead

Matthew Feagan

Darren Fearon

Mike Fedun

Rachel Ann Firlotte

Sara A Fletcher

Susan Flueck
Hudson Foley

Elizabeth A. Francis

Erin Fraser

Kevin Freehill

Dennis Frisky

Dallas Frith

Mark Froehler

Curtis H. Fudge

Glen Gache

Christina Gagnon

William A. Gaines

Chad Gardeski

Randy Gauthier

Christine Gelowitz

Jennifer George

Andrew Gesner Rob Gibb

Jamie R. Giberson

Stephen J. Gill

William Gilmour

Jean Girard

Lauriant Giroux

JeanPaul Rene J Gladu

Dale F. Goodfellow

Tammy L. Goodwin

Shawn Goodyear

Richard Goy

Gordon Graham

Ross Graham

Christopher J Grainger

Chris A Grant

Roger Graves

Conrad Gray

Chris J. Gray

Jennifer Hacking

Peter Haggerty

Kevin A. Hakes

Amanda J. Hamelink

Robert Hamilton

Christina A. Hamilton Mike Hancock

Timothy M Harapiak

Ginger Harke

Donald Harrison

Rick Harrison

Joseph W. Hart

Kevin Heartwell

David Heatherington

Martin Hemsley Jeff Henricks

Howard Herman

Rupert Hewison

Raymond Hilts

Joel M Holder

David Holland

Erik Holmstrom

Richard Horne

Jeffrey Horner Rod Houle

Rhonda Hudson Bill Hudson

Dave Hugelschaffer

Jennifer Hughes

Tjerk Huisman

Kerry S. Hutchings

Jason Jabbour

Hal E. Jackson

Kristin Jensen

Ryan Jillard

Darryl Johnson

Faye J. Johnson

Beau D. Johnson
Caroline Johnston

Iain Johnston

Greg Scott Jonuk

Patrick Jordan

Shelby Jorgenson

Simon Kaderabek

Michael Kakoullis

Nathan Kalinocka

Morgan Kehr

Mary K. Kelly

Kris Kennedy

Marla D. Kimball

Matthew Kinch

Glenn T. King

Kelly Ann King

Michael Kingsbury

Thor Knapp

Faron Knott

Denis LaBonte

David Laing

Mike Landers

Dion Lawrence

Jules Leboeuf

Marcel LeCoure

Kevin Ledieu

Laurier Lefebvre

L. Makenzie Leine

Trevor Lema

William Lesiuk

Trevor LeskeChibry

G David Lind

H. Richard Lipman

Brian Lopushinsky

Liana Luard

Deb Lucas

James Lunn

Hans Lussenburg

Michael Lutz

Warren Mabee

Jason H. MacDonald

John MacDougall

Bruce MacGregor

Greg A. MacKeigan

Lorraine Maclauchlan

M Sean MacNeil

Sheila Madahbee

Scott Maher

Sherry Maine

Jim Maitland

Melanie Makoweck

M. Dawn Manning

Adam Marshall

Wenc Martinek

Erika A. Marzinotto

Sean Mastervick

Max S. Mathon

Gerald (Gerry) Matthews

Larry Matwie

Paul Mayer

Robert McAdam

Bart McAnally

Ken McCrae

Dan McCurdy

Wade McDonald

Gillian E. McEachern

Barry McElhinney

Christopher McGuinty

Rory L. McIntosh

Rebecca McKay

Heather L. McLaren

Tracy McLean

Andrew McLellan 
New Members
for the year 2000

John McLevin

Matthew W. McMillan

Rand McPherson

Roger Meyer

Mark Mill

Kenton Miller

Michael Milner

Eileen Mitchell

Shaun Mitchell

Ryan J. Monette

Rick Moore

Dale R Morgan

Bernard Morin

Duncan Moss

(Grant) Richard Moyse

Rainer Muenter

R. Jason Mullins

Chantal D. Myers

James T. Nash Greg Neale

Doug W Nichol

Roy Nichols

Gunilla Nilsson

Wesley Nimco

Dave Nordquist

Julian M. Norghauer

Leonard Norman

Cathy Norton

Marty O'Byrne

Dollard O'Connor

Aynslie E Ogden

Bryan R. Oke

Steven P. Oldford

Robert Olenick

Ray Olsson

Barrie Onysty

Kenneth Orich

Mike O'Rourke

Derek R. Osborne

Barbara Osterhout

Martha Ann O'Sullivan

Anand Pandarinath

William H. Parker

David F.H. Parker

Michael Pasula

Brad Patrick

Robert Patterson

Hugues D Paulin

Matthew Pawliuk

\section{Karl Peck}

Tyler Pell

Jarmo A. Peltola

Renee L. Perry

Wally Peters

Robert Petite

Tim Phaneuf

Paul Picard

Brad Pinno

Ronald Plinte

Tom Plouffe

Ken Podulsky

Jeff Poeckens

Marc A. Poirier

Kenneth Porter

Richard Prince

Lynn Profitt

Reino Pulkki

Dana Quartly

William Redhead

Stephen Reed

Beatrice M. Regnier

Mark Reiter

Cory Rhett

Kyna A. Rigal

Jason Rilling

Geordie Robere-McGugan

Philippe Robert

Allan Robinson

Patricia Rodseth Pat Rogers

H. Raymond Rolfe

Darryl Rollings

Derrick A. Romain

Paul Ronellenfitch

Michael Robert Rosen

Jean-Claude Ruel

Doug Ruffle

Tony Saint

Darryl Sande

Mandeep Sandhu

Valerie Saunders

Jeff Scammell

Trevor G. Scheers

Brent Schleppe

Bernard Schmitte

Heath Schneider

David K. Schofield

Peter M. Schubert

Claude Schweizer

John T. Scott

Paul W. Secker

\section{Members for 25 Years 2001}

Martin E Alexander

Earl Anderson

Peter Annis

Larry Atherton

Brian Barkley

Rodney Beaumont

Ron Bronstein

Robert G Bull

A Boyd Case

Robin Clark

Mike Clarke

Dusan Dodic

Edward Evans W H Fogal

Warren B Gayle

Andrew M. Gordon M E Guptill
Katherine Hopkins

Robert M Larson

David G Lindenas

Thomas J Lundgren

Peter MacQuarrie

Peter Marshall

Leo J O'Driscoll Dean Raven

Richard Rothwell

Hugh Schooley

Kenneth Snow

Robert J. Stoddart

Brad J. Sutherland Jordan Tanz

Allan J. Willcocks

Evelynne M Wrangler Cheng Ying
Lloyd Seedhouse

Carris Serben

Michael Setterington

Alastair Shenfield

Kelly Sherman

James William Short

Juan D. Silva

Francis Skeard

Darryl Slater

Chris Smith

Evert Smith

Inga Nancy Ann Smith

Rickey Smith

Eryn Smith

Kenneth Snyder Clint Soltys

Jamie B. Somerville

Ross Spence

Cameron Spry

Paul St John

Bogoljub Stankovic

Brian Stanton

Melissa Steidle

Paul Steiestol

Frank Steil

Cindy Stern

Richard Stewart

Tracey Stewart

Mark Storie

Len Stroebel

Thom E. Stubbs

Margaret Symon

Shereen Tamara Taylor

David W. Taylor

David C. Taylor

Gordon Taylor John Taylor

Doug C. Taylor Don Taylor

James L Taylor

Roger Tetreault

Victor Theoret

Evelynne Thiffault

Dale Thomas

Doug Thompson

Michael Thompson

Rob Thorburn

Christie Thorburn

William Thresher Bill Tinge

Victor Toutant

Tim W. Trahan

\section{Year Members 2001}

Thomas W B Bartlett

Paul E Boucher

Robert J Halliday

Edward L Hughes

Henry J Johnson

Samuel N Linzon

Thomas H Lloyd

Paul G Masterson
Edward Oliver Trenchard

Chris Trudel

Gail Tucker

Keith Tucker

Jennifer Turner

Richard Ubbens David Usher

Kari E. Van Allen Lori Vance

William Vandyk

Kirsten Vice

Norman Volk

Patricia Von Ahn

Albert Walker

Dave Wallace

Ian Wallis

Chris Walsh

Jian Rang Wang

Michael Watson

Leslie Weekes

Jacquelyn M. Wells

Brian Wesolowsky David West

Kevin Westerhaug

Michael Weyer

Thomas White

Justin B. Whitney Aprile Whyte

Wayne Williams

Willy $\mathrm{M}$. Wilondja

Leonard Wilton

Axel Winter Brian Wog

Cory Wojtowicz Kyle Wolfe

Robert G. Wood

Nikki L. Wood

Michael Woods

Ralph Woods

Blair Wriglesworth

Therese Yacyshyn Dennis York

Robert Yowney

Chantal Zimmer

Dmitri Zvonka

Shaun Zwerzinski 